câu là 56\%.

Bệnh nhân không ho ra máu $(73,3 \%)$ có kết quả điêu trị tốt nhiều hơn rõ rệt so với bệnh nhân có ho ra máu $(6,7 \%)$, với $p<0,05$. Bệnh nhân XQ Phổi không có hang $(63,3 \%)$ có kết quả điêuu trị tốt nhiều hơn rõ rệt so với bệnh nhân XQ Phổi có hang $(16,7 \%)$, với $p<0,05$. Bệnh nhân HIV (-) $(85 \%)$ có kết quả điều trị tốt nhiều hơn so với bệnh nhân HIV $(+)(13,3 \%)$, với p> 0,05, chưa có ý nghĩa thống kê.

Bệnh nhân khám phổi có ran $(18,3 \%)$ có kết quả điều trị kém nhiều hơn rõ rệt so với bệnh nhân khám phổi không có ran $(1,7 \%)$, với $\mathrm{p}<$ 0,01 . Bệnh nhân có AFB (+) $(18,3 \%)$ có kết quả điều trị kém nhiều hơn so với bệnh nhân có $A F B$ (-) $(1,7 \%)$, với $p>0,05$, chưa có ý nghĩa thống kê. Bệnh nhân thể lao cũ $(13,3 \%)$ có kết quả điêuu trị kém nhiêuu hơn so với bệnh nhân thể lao mới (3,3\%), với p>0,05. Một số nghiên cứu cũng xác định liên quan với kết quả điều trị kém là: xét nghiệm soi đờm tìm AFB (+) là yếu tố nguy cơ độc lập (OR hiệu chỉnh = 1,94) [3]; hang lao (OR, 0,175; CI 95\%, 0,108-0,286, p<0,001) và thời gian điêu trị lao trênmột năm $(O R, 0,077$; 95\%CI, 0,011-0,499, p<0,001) [2].

\section{KẾT LUÂN}

Bệnh nhân RR-TB thường gặp nhiều ở nhóm tuổi trung niên, thể lao cũ, soi đờm có AFB (+), tổn thương Xquang Phổi rộng. Các yếu tố ảnh hưởng đến kết quả điều trị kém là: thể lao cũ, khám phổi có ran, soi đờm có AFB $(+)$, Xquang Phổi có hang.

\section{TÀI LIỆU THAM KHẢO}

1. Bô Y tế, (2018), Hướng dẫn chẩn đoán, điều trị và dự phòng bệnh lao, Quyêt định số 3126/Q' - BYT.

2. Liu CH, Li L, Chen $Z$, Wang $Q$, Hu $Y L$, et al. (2011), Characteristics and Treatment Outcomes of Patients with MDR and XDR Tuberculosis in a TB Referral Hospital in Beijing: A 13-Year Experience. PLoS ONE 6(4): e19399. doi:10.1371/ journal. pone.0019399.

3. Monadil H Ali, et al.2019, "Assessment of multidrug-resistant tuberculosis (MDR-TB) treatment outcomes in Sudan; findings and implications", pp 6.

4. Orina GM, et al., (2017), Comparative Study of Smear Microscopy, Gene Xpert and Culture and Sensitivity Assays in Detection of Mycobacterium tuberculosis on Sputum Samples among Tuberculosis Suspected Cases in Nyamira County Referral Hospital, University Nairobi, Kenya, Mycobact Dis 2017, 7:3 DOI: 10.4172/21611068.1000249.

5. Tang S, Tan S, Yao L, Li F, Li L, et al. (2013), Risk Factors for Poor Treatment Outcomes in Patients with MDR-TB and XDR-TB in China: Retrospective Multi-Center Investigation. PLoS ONE 8(12): e82943.doi:10.1371/journal.pone.0082943

6. WHO (2019), Global tuberculosis report 2019, pp 3,58 .

\title{
KHẢO SÁT TƯƠNG QUAN GIỮA KHOẢNG CÁCH GIỮA HAI ĐỒNG TỬ VỚI KHOẢNG CÁCH GIŨ̃A HAI GÓC MẮT TRONG Ở NGƯờI VIÊT TRƯỞNG THÀNH - NGHIÊN CỨU SỬ DỤNG TRONG PHỤC HÌNH Ổ MẮT
}

\section{TÓM TẮT}

Mục tiêu: Khảo sát tương quan giữa khoảng cách giữa hai đồng tử với khoảng cách giữa hai góc mắt trong ở người Việt trưởng thành, giúp cung cấp thêm thông tin cho việc thức hiên phục hình ổ mắt, một loai hình trong phục hình hàm mặt. Đối tượng và phương pháp: Nghiên cứu cắt ngang mô tả qua ảnh chụp mặt thẳng của 100 sinh viên khoa Răng Hàm Mặt - Đại học Y Dược thành phố Hồ Chí Minh, tuổi tù̀ 21 đến 28. Dùng phần mềm AutoCAD để đo các kích thước trên ảnh và phân mềm SPSS để phân tích thống kê. Kết quả: Nghiên cứu cho thấy khoảng cách giữa

${ }^{1}$ Đai hoc Y Dước TP. Hồ Chí Minh

Chịu trách nhiệm chính: Nguyễn Thái Phượng

Email: ntphuong@ump.edu.vn

Ngày nhận bài: 18/1/2021

Ngày phản biên khoa học: 21/2/2021

Ngày duyệt bài: 5/3/2021

\section{Nguyễn Thái Phượng ${ }^{1}$ Đoàn Minh Trí}

hai đồng tử có tương quan mạnh với khoảng cách giữa hai góc mắt trong khi xét trên toàn bộ mấu lẫn khi phân chia theo giới $(r>0,5)$. Sử dụng phương trình hồi quy tuyến tính đơn biến giúp dự đoán khoảng cách giữa hai đồng tử từ khoảng cách giữa hai góc mắt trong. Kết luâan: Khoảng cách giữa hai đồng tử có tương quan mạnh với khoảng cách giữa hai góc mắt trong. Có thể dự đoán sơ khởi khoảng cách giữa hai đồng tử từ khoảng cách giữa hai góc mắt trong bằng phương trình hồi quy tuyến tính đơn biến.

Tư khóa: Khoảng cách giữa hai đồng tử, khoảng cách giữa hai góc mắt trong, phục hình ổ mắt.

\section{SUMMARY}

EVALUATION OF THE CORRELATION BETWEEN INTERPUPILLARY DISTANCE AND INTERCANTHAL DISTANCE IN THE ADULT VIETNAMESE - THE STUDY USING FOR OCULAR PROSTHESIS

Objectives: Evaluating the correlation between 
interpupillary distance and intercanthal distance in the adult Vietnamese helps to provide more information using for ocular prosthesis, one kind of maxillofacial prostheses. Subjects and method: The crosssectional study with 100 Vietnamese dental students who ranged from 21 to 28 years of age. Standardized digital images from the frontal aspect of the faces were taken and AutoCAD software was used to measure these dimensions. The data were statistically analyzed by SPSS software. Results: The study founded that having a strong correlation between interpupillary distance and intercanthal distance following all subjects or gender dividing $(r>0,5)$. The monovariant linear regression equations were used to predict interpupillary distance from intercanthal distance. Conclusion: The interpupillary distance has been significantly correlated with intercanthal distance. The intercanthal distance can be used as a preliminary method for estimating the interpupillary distance by using the monovariant linear regression equations.

Keywords: Interpupillary distance, intercanthal distance, ocular prosthesis.

\section{I. ĐẶT VẤN ĐỀ}

Mắt là trung tâm biểu lô cảm xúc con người. Việc mất mắt do tai nạn, bệnh lý phải phẫu thuật lấy nhãn cầu hay trong một số dị tật thiếu mắt bẩm sinh thường để lại những di chứng nặng nề cả về thẩm mỹ lẫn tâm lý cho bệnh nhân. Phục hình trong ổ mắt (ocular prosthesis) là loai phục hình hàm mặt giúp phục hồi lại vẻ ngoài và sự tự tin, nhằm nâng cao chất lượng cuộc sống cho bệnh nhân. Khi thực hiện phục hình ổ mắt, bác sĩ cần xác định vị trí đồng tử bên mắt bị mất giúp phục hình có sự hài hòa với mắt còn lại.

Vì vậy, khoảng cách giữa hai đồng tử là một thông số quan trọng giúp thực hiện thành công phục hình mắt làm sẵn (phục hình mắt tạm) hay trong phục hình mắt sau cùng. Ngoài ra, kích thước này còn có ý nghĩa giúp việc chẩn đoán và điêu trị các bệnh lý và hội chứng sọ mặt. Vì vậy, các nhà lâm sàng trên thế giới đã có những nghiên cứu đo đạc khoảng cách giữa hai đồng tử như nghiên cứu của Pryor [7], Jai Rup Singh [4], Farkas [1], Murphy [5], Osuobeni [6], Razavi [8], Hayat [3]... Một số nghiên cứu còn đánh giá khoảng cách giữa hai đồng tử trong mối tương quan với các kích thước răng, nhằm tìm ra thông số đáng tin cậy hướng dẫn cho việc chẩn đoán, điều trị và thực hiện phục hình răng như nghiên cứu của Gomes [2].

Khi đã phẫu thuật cắt bỏ một mắt, việc xác định khoảng cách giữa hai đồng tử gặp nhiều khó khăn. Để ước tính giá trị này, một số tác giả đề nghị dựa vào khoảng cách giữa hai góc mắt trong và khoảng cách giữa hai góc mắt ngoài như Pryor [7] và Jai Rup Singh [4]. Trong khi đó, Osuobeni tìm thấy mối tương quan mạnh giữa khoảng cách giữa hai đồng tử với khoảng cách giữa hai góc mắt trong [6]. Tuy nhiên, các dữ liệu về mối quan hệ này khá hiếm trong y văn cũ̃ng như kết quả có thể khác biệt theo từng chủng tộc hay quốc gia. Vì vây, chúng tôi muốn tiến hành nghiên cứu thăm dò nhằm tìm ra đặc điểm phù hợp cho người Việt Nam, trên cơ sở ứng dụng để thực hiện phục hình ổ mắt.

Đề tài được thực hiện nhằm mục tiêu xác định khoảng cách giữa hai đồng tử và khoảng cách giữa hai góc mắt trong cũng như phân tích sự khác biệt về hai thông số trên giữa nam và nữ; từ đó xác định tương quan giữa khoảng cách giữa hai đồng tử với khoảng cách giữa hai góc mắt trong; đồng thời đưa ra phương trình hồi quy tuyến tính đơn biến giúp dự đoán sơ khởi khoảng cách giữa hai đồng tử từ khoảng cách giữa hai góc mắt trong nếu tương quan ý nghĩa.

\section{II. ĐỐI TƯỢNG VÀ PHƯƠNG PHÁP NGHIÊN CỨU \\ 2.1. Đối tượng nghiên cứu}

Cõ̃ mẫu được xác định bởi công thức áp dụng cho các nghiên cứu ước tính hệ số tương quan:

$$
n=3+\frac{C}{0.25\left[\log \left(\frac{1+r}{1-r}\right)\right]^{2}}
$$

Trong đó: $\mathrm{n}$ là cõ̃ mẫu nghiên cứu cần có, $\mathrm{C}$ là hằng số liên quan đến sai sót loại I và II $(\mathrm{C}=$ $10,51$ khi chọn $a=0,05$ và $\beta=0.1)$, $r$ là hệ số tương quan quan sát được (chọn $r=0,5$ theo nghiên cứu của Osuobeni khi khảo sát trên nam người Ả rập Saudi). Chúng tôi tính được cỡ mẫu là 38 đối tượng. Thực tế chúng tôi chọn 50 đối tượng cho nam và 50 cho nữ.

Như vậy, mẫu nghiên cứu gồm 100 sinh viên (50 nam và 50 nữ) khoa Răng Hàm Măt - Đại học Y Dược thành phố Hồ Chí Minh, tuổi từ 21 đến 28 , đồng ý tham gia nghiên cứu và thỏa các điều kiện sau: có cha mẹ là người Việt Nam; có khuôn mặt bình thường; không có dị dạng mặt bẩm sinh, bệnh về mắt, tiền sử chấn thương hay phẫu thuật mặt.

\subsection{Phướng pháp nghiên cứu}

- Thiết kế nghiên cứu: Đây là nghiên cứu cắt ngang mô tả.

- Phương tiênn nghiên cứu: Bút lông kim đầu nhỏ. Máy ảnh kỹ thuật số Canon, loại SLR, hiệu EOS 300D. Máy tính với phần mềm đo đạc AutoCAD 2016. Thước nhân trắc, thước thủy tĩnh. Hệ thống chụp ảnh khuôn mặt với giá cố định đầu của máy chụp phim đo sọ.

\section{- Các bước tiến hành}

Bước 1: Chụp ảnh khuôn mặt nhìn thẳng của đối tượng ở tư thế nghỉ sinh lý theo các tiêu chuẩn:

- Đối tượng ngồi trên ghế, mắt nhìn thẳng 
theo đường ngang, thả lỏng cơ mặt miệng, thư giãn hàm dưới và hai môi chạm nhẹ.

- Hai nút tai của giá cố định đầu được cài vào lỗ ống tai ngoài của đối tượng, chỉnh sao cho ba điểm: bờ trên của lỗ ống tai ngoài $(\mathrm{Po})$, điểm dưới ổ mắt (Or), điểm định vị phía mũi của máy chụp $X$ quang nằm trên một đường thẳng. Như vậy, chúng tôi xác định được mặt phẳng Frankfort của đối tượng song song với mặt phẳng ngang và đầu đối tượng được giữ yên ở một vị trí (Hình 1).

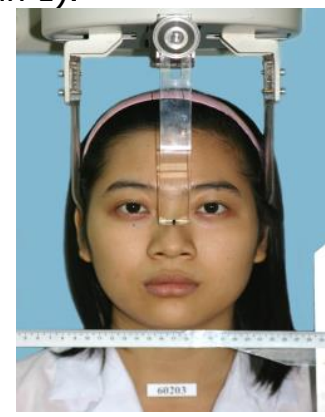

Hình 1. Chụp ảnh khuôn mặt nhin thẳng

Bước 2: Chuyển hình ảnh vào máy vi tính.

Bước 3: Xác định các điểm chuẩn (Hình 2):

- P: Tâm đồng tử

- En: Điểm nằm ở khóe trong của rãnh mi mắt

Bước 4: Dùng phần mềm AutoCAD đo đạc (Hình 2):

- Khoảng cách giữa hai đồng tử (ký hiệu KĐT): là chiêu dài P-P.

- Khoảng cách giữa hai góc mắt trong (ký hiệu KGMT): là chiều dài En-En.

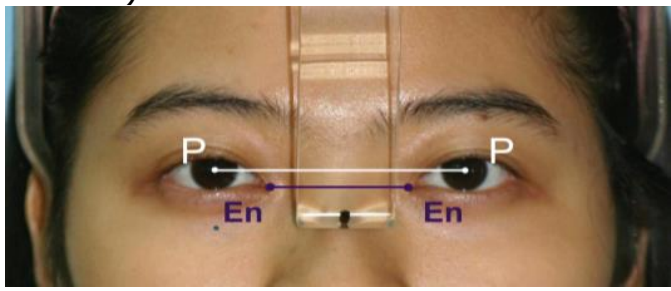

Hinh 2. Đo đạc các số đo ở vùng mắt

Các ảnh đều được chụp và đo đạc bởi tác giả chính. Đồng thời, khoảng cách từ ống kính đến đối tượng, ánh sáng, tiêu cự đều giống nhau trong các lần chụp.

- Xử lý số liệu: Dùng phần mềm thống kê SPSS 16.0 để nhập và xử lý số liệu: Tính trung bình và độ lệch chuẩn các giá trị đo đạc. Dùng kiểm định $\mathrm{t}$ cho hai mẫu độc lập để so sánh các giá trị đo đạc giữa nam và nữ. Dùng hệ số tương quan Pearson để đánh giá tương quan giữa khoảng cách giữa hai đồng tử với khoảng cách giữa hai góc mắt trong. Lập phương trình hồi quy thể hiện tương quan đồng tử - mắt (nếu có tương quan ý nghĩa). Dùng kiểm định $\mathrm{t}$ bắt cặp để so sánh giá trị tiên đoán và giá trị đo thực tế.

- Đạo đức trong nghiên cứu: Đối tượng nghiên cứu được giải thích rõ về mục đích nghiên cứu, trách nhiệm của người nghiên cứu, trách nhiệm và quyền lợi của người tham gia nghiên cứu. Nghiên cứu chỉ được tiến hành trên những đối tượng tự nguyện tham gia. Toàn bộ thông tin thu thập được bảo mật và chỉ phục vụ cho mục đích nghiên cứu.

\section{KẾT QUẢ NGHIÊN CỨU}

Qua khảo sát 100 người Việt Nam (gồm 50 nam và 50 nữ) tuổi từ 21 đến 28 với những đặc điểm nghiên cứu đều tuân theo luật phân phối chuẩn, chúng tôi ghi nhận một số kết quả sau:

3.1. Khoảng cách giữa hai đồng tử và khoảng cách giữa hai góc mắt trong

3.1.1. Xét trên toàn bô mẫu. Các giá trị trung bình, độ lệch chuẩn, giá trị lớn nhất, giá trị nhỏ nhất được trình bày tại bảng 1.

Bảng 1. Kêt quả đo đạc các kích thước vùng mắt trên toàn bộ mẫu $(n=100)$

\begin{tabular}{|c|c|c|c|c|}
\hline $\begin{array}{c}\text { Kích thước } \\
(\mathbf{m m})\end{array}$ & TB & ĐLC & GTNN & GTLN \\
\hline KĐT & 63,21 & 3,06 & 56,01 & 71,52 \\
KGMT & 35,28 & 2,28 & 28,49 & 40,59 \\
\hline
\end{tabular}

3.1.2. Xét theo giới. Khi so sánh giữa nam và nữ, khoảng cách giữa hai đồng tử khác biệt ở hai giới có ý nghĩa thống kê $(p<0,05)$, cụ thể, giá trị trung bình của nam lớn hơn của nữ. Tuy nhiên, khoảng cách giữa hai góc mắt trong chưa thấy có sự khác biệt theo giới (Bảng 2).

Bảng 2. Kêt quả đo đạc các kích thước vùng mắt theo giới

\begin{tabular}{|c|c|c|c|}
\hline $\begin{array}{l}\text { Kích } \\
\text { thước } \\
\text { (mm) }\end{array}$ & $\begin{array}{c}\text { Nam }(n=50) \\
\text { TB } \pm \text { ĐLC }\end{array}$ & $\begin{array}{l}\text { Nũ̃ }(n=50) \\
\text { TB } \pm \text { ĐLC }\end{array}$ & $\mathbf{p}^{*}$ \\
\hline KФT & & & \\
\hline KGM1 & $35,56 \pm 2,34$ & $\pm 2,21$ & 0,22 \\
\hline
\end{tabular}

*Kiểm định $\mathrm{t}$ cho hai mầu độc lập

3.2. Tương quan giữa khoảng cách giữa hai đồng tử với khoảng cách giữa hai góc mắt trong. Kết quả cho thây khoảng cách giữa hai đồng tử có tương quan mạnh với khoảng cách giữa hai góc mắt trong, khi xét trên tổng mẫu lẩn khi phân chia theo giới $(r>0,5)$ (Bảng 3).

Bảng 3. Tương quan giữa khoảng cách giữa hai đồng tử với khoảng cách giữa hai góc mắt trong

\begin{tabular}{|c|c|c|c|c|c|c|}
\hline \multirow{2}{*}{ KGMT } & \multicolumn{2}{|c|}{ Toàn bộ } & \multicolumn{2}{c|}{ Nam } & \multicolumn{2}{c|}{ Nữ } \\
\cline { 2 - 7 } & $\mathrm{r}$ & $\mathrm{p}$ & $\mathrm{r}$ & $\mathrm{p}$ & $\mathrm{r}$ & $\mathrm{p}$ \\
\hline KĐT & 0,558 & 0,000 & 0,627 & 0,000 & 0,589 & 0,000 \\
\hline
\end{tabular}

- r:Hệ số tương quan Pearson; p: Mức ý nghĩa

Từ đó, chúng tôi đưa ra phương trình hồi quy tuyến tính đơn biến giúp dự đoán sơ khởi 
khoảng cách giữa hai đồng tử từ khoảng cách giữa hai góc mắt trong (Bảng 4).

Bảng 4. Phương trinh hồi quy thể hiện tương quan giữa khoảng cách giữa hai đồng tư với khoảng cách giữa hai góc mắt trong

\begin{tabular}{|c|c|c|}
\hline Toàn bộ & Nam & Nữ \\
\hline$y=0,790 x$ & $y=0,771 x$ & $y=0,665 x$ \\
$+35,337$ & $+37,293$ & $+38,452$ \\
\hline
\end{tabular}

$x$ : Khoảng cách giữa hai góc mắt trong; $y$ : Khoảng cách giữa hai đồng tử

Dùng kiểm định $\mathrm{t}$ bắt cặp để so sánh giá trị tiên đoán theo phương trình hồi quy với giá trị đo thực tế, kết quả cho thấy sự khác biệt không có ý nghĩa thống kê $(p>0,05)$.

\section{BÀN LUÂ̂N}

4.1. Vê khoảng cách giữa hai đồng tử. Là kích thước từ tâm đồng tử bên phải đến tâm đồng tử bên trái. Đây là mốc giải phẫu tốt nhất về khoảng cách giữa trung tâm hai nhãn câu [7]. Lucas năm 1935 nhận xét thông số này sẽ không tăng thêm sau 14 tuổi [2]. Nhìn chung, các tác giả đo đạc theo hai phương pháp chính: đo đạc trực tiếp bằng thước kẹp [5], thước nhựa [3], dụng cụ đo mắt như $\mathrm{PD}$ metre [8] hay auto refractometer [3]; hoặc gián tiếp thông qua ảnh chụp $[2,6]$.

Trong nghiên cứu này, khoảng cách giữa hai đồng tử có giá trị trung bình khoảng $63 \mathrm{~mm}$, xấp xỉ các nghiên cứu trên thế giới $[3,5]$. Đồng thời, chúng tôi nhận thấy có sự khác biệt có ý nghĩa thống kê giữa nam và nữ; tương tự như nghiên cứu của Murphy [5], Gomes [2] và Hayat [3].

4.2. Về khoảng cách giữa hai góc mắt trong. Vị trí gặp nhau của hai mí mắt gọi là góc mắt. Khoảng cách giữa hai góc mắt trong được đo giữa hai điểm ở góc mắt trong của rãnh mí mắt phải và trái. Theo Farkas, khoảng cách này đạt $84,1 \%$ trong năm đầu tiên, sau đó tăng lên $93,3 \%$ lúc 5 tuổi và $100 \%$ khi 8 đến 11 tuổi [1]. Khoảng cách giữa hai góc mắt trong có nhiêu ứng dụng quan trọng trong y khoa như giúp đánh giá các mức độ chấn thương hay hỗ trợ chẩn đoán, điều trị sớm một số hội chứng sọ mặt bẩm sinh, đồng thời lập kế hoạch tạo hình sửa chữa những khiếm khuyết bẩm sinh hay mắc phải [5].

Nghiên cứu cho thấy khoảng cách giữa hai góc mắt trong có giá trị xấp xỉ người Brazil [2] và người da đen Mỹ [5] nhưng khá lớn so với người da trắng Mỹ [7], người Ấn Độ [4] và Pakistan [3]. Đồng thời, số đo này khồng khác biệt giữa nam và nữ, phù hợp với phát biểu của Gomes [2] nhưng trái ngược với Murphy [5] và Hayat [3].
4.3. Vê tương quan giữa khoảng cách giữa hai đồng tữ với khoảng cách giữa hai góc mắt trong. Năm 1969, Pryor đề nghị một phương pháp đơn giản để ước tính khoảng cách giữa hai đồng tử từ khoảng cách giữa hai góc mắt trong và khoảng cách giữa hai góc mắt ngoài, bởi việc đo đạc trực tiếp đồng tử ở trẻ em là điều hết sức khó khăn [7]. Feingold năm 1974 và Jai Rup Singh năm 1983 cũng trình bày mối liên quan giữa các thông số trên bằng phương trình hồi quy đa biến [4].

Năm 1993, Osuobeni khi nghiên cứu 198 nam người Ả rập Saudi với độ tuổi từ 16 đến 25 đã tìm thấy mối tương quan mạnh giữa khoảng cách giữa hai đồng tử với khoảng cách giữa hai góc mắt trong với hệ số tương quan $r=0,55$ [6]. Tuy nhiên, các dữ liệu có thể khác biệt theo từng chủng tộc hay quốc gia. Razavi năm 2008 khảo sát trên trẻ em Iran nhưng không tìm được công thức thể hiện sự tương quan giữa hai thông số trên [8].

Trong nghiên cứu này, chúng tôi đánh giá mối tương quan giữa khoảng cách giữa hai đồng tử với khoảng cách giữa hai góc mắt trong, bởi cấu trúc này tương đối ổn định trong trường hợp phẫu thuật cắt bỏ mắt không quá xâm lấn (kỹ thuật Evisceration). Kết quả cho thấy khoảng cách giữa hai đồng tử có tương quan mạnh với khoảng cách giữa hai góc mắt trong, khi xét trên tổng mẫu lẫn khi phân chia theo giới $(r>0,5)$. Từ đó, chúng tôi đề nghị dùng khoảng cách giữa hai góc mắt trong để dự đoán sơ khởi khoảng cách giữa hai đồng tử bằng phương trình hồi quy tuyến tính đơn biến.

\section{KẾT LUẬN}

Đây là nghiên cứu thăm dò về tương quan giữa khoảng cách giữa hai đồng tử với khoảng cách giữa hai góc mắt trong ở người Việt Nam trưởng thành. Dù đối tượng sinh viên chưa đại diện cho toàn bộ dân số, nghiên cứu cũng góp thềm tư liệu giúp thực hiện phục hình ổ mắt sao cho hài hò̀a, tự nhiển và thẩm mỹ, nhằm nâng cao chất lượng cuộc sống của bệnh nhân.

\section{TÀl LIÊU THAM KHẢO}

1. Farkas LG, Posnick JC, Hreczko TM, Pron GE. "Growth patterns in the orbital region: a morphometric study". Cleft Palate Craniofac J, 1992, 29(4):315-8.

2. Gomes VL., Gonçalves LC., do Prado CJ., Junior IL., Lucas Bde L. "Correlation between facial measurements and the mesiodistal width of the maxillary anterior teeth". J Esthet Restor Dent, 2006, 18(4):196-205.

3. Hayat $\mathbf{N}$. "Normal interpupillary, inner canthal distance and outer canthal distance in a normal 
population of Pakistan". Pak J Med Sci, 2019, 35(1):50-54.

4. Jai Rup Singh, Sarla Banerjee. "Normal value for interpupillary, inner canthal and outer canthal distances in an Indian population". Human Heredity, 1983, 33(5):326-328.

5. Murphy KW., Laskin DM. "Intercanthal and interpupillary distance in the black population". Oral Surg Oral Med Oral Pathol, 1990, 69:676-80.
6. Osuobeni EP, Al-ibraheem AM. "Ocular and facial dimensions of male Arab children". J Am Optom Assoc, 1993, 64:717-7.

7. Pryor HB. "Objective measurement of interpupillary distance". Pediatrics, 1969, 44:973-977.

8. Razavi ME, Jalalifar S. "Correlation between Interpupillary and Inner-Outer Intercanthal Distances in Individuals Younger than 20". ] Ophthalmic Vis Res, 2008, 3(1):16-22.

\section{KIẾN THỨC CỦA ĐIỀU DƯỡNG VỀ PHÒNG NGỪA TÉ NGÃ CHO NGƯỜI BỆNH TẠI BỆNH VIỆN ĐA KHOA TỈNH NAM ĐỊNH NĂM 2020}

\section{TÓM TẮT}

Mục tiêu: Mô tả thực trạng kiến thức của điều dưỡng vềphòng ngừa té ngã cho người bệnh tại Bệnh viện Đa khoa tỉnh Nam Định Đối tượng và phướng pháp nghiên cứu: Nghiên cứu mô tả cắt ngang vớl sư tham gia của 170 điều dưỡng trực tiếp chăm sóc người tại các khoa lâm sàng Bệnh viện Đa khoa tỉnh Nam Định từ tháng 12/2019 đến tháng 6/2020. Kết quả: Đa số điều dưỡng là nữ giới $(81,8 \%)$ và có thâm niên công tác dưới 10 năm chiếm tỷ lê cao nhất (51,8\%). Điều dưỡng có số người bệnh phải chăm sóc trung bình/ ngày dưới 10 người bểnh là $(58,8 \%)$ và vẫn còn $34,7 \%$ chưa tham gia các lớp đào tạo về phòng ngừa té ngã. Tỷ lệ điều dưỡng có kiến thức về phòng ngừa té ngã cho người bệnh ở mức đạt là $56,5 \%$. Kết luân: Mức độ kiến thưư phòng ngửa té ngã cho người bệnh của điều dưỡng chưa thực sự cao. Kết quả nghiên cứu cho thây sự cần thiết phải củng cố kiến thức cho điêu dưỡng về dự phòng té ngã cho người bênh.

Tứ khoá: Kiến thức, phòng ngừa té ngã, người bệnh, điều dưỡng

\section{SUMMARY}

NURSES' KNOWLEDGE ON FALL PREVENTATION FOR PATIENTS AT

\section{NAMDINH GENERAL HOSPITAL 2020}

Objective: Describe the nurses' knowledge on fall preventation for patients at Namdinh General Hospital. Subjects and methods: A cross-sectional study on 170 nurses who directly care for patients at clinical units at Namdinh General Hospital during 12/2019 and 6/2020. Results: The majority of nurses are women $(81.8 \%)$ and have been working for less than 10 years, accounting for the highest percentage (51.8\%). The average number of nurses taking care of less than

${ }^{1}$ Trường ĐH Điều dưỡng Nam Định Chiu trách nhiêm chính: Hoàng Thị Minh Thái Email: minhthai82nd@gmail.com Ngày nhận bài: 2/1/2021

Ngày phản biện khoa học: 1/2/2021

Ngày duyệt bài: 3/3/2021
Hoàng Thị Minh Thái ${ }^{1}$, Đinh Thị Thu Hằng ${ }^{1}$, Phạm Thị Bích Ngọc ${ }^{1}$, Vũ Thị Lài , Vũ Thị Én'

10 patients per day is (58.8\%) and $34.7 \%$ have not yet attended training courses on falls prevention. The percentage of nurses, who had sufficient knowledge, was $56,5 \%$. Conclusion: The nurses' knoledge and practice on fall preventation for patients were limited. Therefor, nurse should be provided knowldge on fall preventation for patients.

Keywords: Knowldge, fall Preventation for patients, nurse, patient.

\section{I. ĐẶT VẤN ĐỀ}

Té ngã là một trong sáu sự cố y khoa được phân loại dựa vào đặc điểm chuyên môn theo Hiệp hội An toàn người bệnh Thế giới. Mỗi năm có khoảng 37,3 triệu ca té ngã cần đến sự chăm sóc y tế, dẫn đến 646.000 ca tử vong do té ngã trên toàn cầu. Té ngã là nguyên nhân đứng hàng thứ hai gây tử vong do thương tích không chủ ý sau tai nạn giao thông đường bộ và thường gặp ở những người từ 65 tuổi trở lên. Tại Việt Nam, theo một thống kê tại Bệnh viện Đại học Y Dược thành phố Hồ Chí Minh năm 2017, té ngã chiếm 92,31\% trong các sự cố $Y$ khoa thường có trong bệnh viện [1]. Té ngã mang lại hậu quả to lớn về không chỉ đến sức khỏe mà còn tác động xấu đến chi phí điều trị cho người bệnh. Với đặc thù nghề nghiệp của mình, Điều dưỡng có thời gian chăm sóc người bệnh so với các đối tượng khác cao hơn nên việc đảm bảo an toàn cho người bệnh nói chung và phòng ngừa té ngã cho người bệnh nói riêng là nhiệm vụ quan trọng. Thông tư số 19/2013/TT-BYT của Bố $Y$ tế đã nêu rõ phòng ngừa người bệnh té ngã là một trong những nội dung khi thiết lập chương trình và xây dựng các quy định cụ thể bảo đảm an toàn người bệnh và nhân viên y tế.

Tại Nam Định, trong những năm qua, ngành Y tế Nam Định đã thực hiện các giải pháp nhằm nâng cao chất lượng dịch vụ khám chữa bệnh. Tuy nhiên, các đánh giá về công tác phòng ngừa 